\title{
MicroRNA-133b is regulated by TAp63 while no gene mutation is present in colorectal cancer
}

\author{
YIFEI CHEN ${ }^{1,2^{*}}$, YI ZHANG ${ }^{3 *}$, JIANHUAI HE ${ }^{4}$, YING FU ${ }^{1,5}$, CHANGWEI LIN $^{1}$ and XIAORONG LI ${ }^{1}$ \\ ${ }^{1}$ Department of Gastrointestinal Surgery, The Third Xiang Ya Hospital of Central South University, Changsha, Hunan 410013; \\ ${ }^{2}$ Class 1 Grade 2012, The Five-Year Program in Clinical Medicine, School of Medicine, Hunan Normal University, \\ Changsha, Hunan 410013; ${ }^{3}$ Department of Oncological Surgery, Affiliated Hospital of Xuzhou Medical University, \\ Xuzhou, Jiangsu 221000; ${ }^{4}$ Department of Breast, Thyroid and Vascular Surgery, Chenzhou No. 1 People's Hospital, \\ Chenzhou, Hunan 423000; ${ }^{5}$ Grade 2012, The Five-Year Program in Clinical Medicine, \\ Xiangya School of Medicine, Central South University, Changsha, Hunan 410013, P.R. China
}

Received June 29, 2016; Accepted August 8, 2016

DOI: $10.3892 /$ or.2017.5371

\begin{abstract}
Downregulation of miR-133b has been reported in multiple types of malignancies including colorectal cancer (CRC). We previously confirmed that TAp63 actively translates microRNA-133b (miR-133b) transcripts. While the presence of miRNA mutations have frequently been described in CRC, most CRCs do not show any variation in the miR-133b coding sequence. Therefore, it is important to elucidate the relationship between TAp63 and miR-133b, and identify other mediators of miR-133b downregulation in CRC. The expression of TAp63 was detected by RT-qPCR, western blotting, immunohistochemistry (IHC) and densitometric analysis using Image-Pro Plus 6.0 software in $38 \mathrm{CRC}$ and corresponding non-cancerous tissues (NCTs). The expression of mature miR-133b was determined by RT-qPCR, in situ hybridization (ISH) and densitometric analysis using Image-Pro Plus 6.0 software. The DNA from 38 CRC tissues and NCTs were screened for miR-133b mutations through sequence analysis. Compared with the NCTs, TAp63 mRNA expression was significantly lower in 21 (55.27\%) tumor tissues. Compared with the NCTs, the miR-133b expression level was significantly lower in 31 (81.58\%) tumor tissues. The expression of miR-133b was found to be positively correlated with TAp63. Loss of TAp63 and miR-133b was associated with an increased likelihood of metastatic events. The area under the ROC curve (AUC) of TAp63 for CRC was 0.623 [95\% confidence interval (CI), 0.497-0.748; $\mathrm{P}=0.046]$, with
\end{abstract}

Correspondence to: Professor Xiaorong Li or Dr Changwei Lin, Department of Gastrointestinal Surgery, The Third XiangYa Hospital of Central South University, Tongzipo Road, Changsha, Hunan 410013, P.R. China

E-mail: lixiaorong@medmail.com.cn

E-mail: linchangwei_1987@sina.com

*Contributed equally

Key words: TAp63, microRNA-133b, colorectal cancer, metastasis
$73.7 \%$ sensitivity and $50 \%$ specificity, respectively. The AUC of miR-133b for CRC was 0.857 (95\% CI, 0.774-0.940; $\mathrm{P}<0.0001$ ), with $78.9 \%$ sensitivity and $81.6 \%$ specificity, respectively. The combined AUC of TAp63 and miR-133b for CRC was 0.881 (95\% CI, 0.805-0.956; P<0.0001), with 89.5\% sensitivity and $71.1 \%$ specificity, respectively. Point mutations within the seed region of miR-133b were found in 1 patient, but the point mutation did not impact the secondary structure of the pre-miR-133b. Therefore, downregulation of TAp63 may be one reason for the dysregulation of miR-133b in CRC. The expression analysis of TAp63 and miR-133b revealed that they may be used as valuable prognostic biomarkers for CRC.

\section{Introduction}

Colorectal cancer (CRC) is a leading cause of cancer-related deaths worldwide (1). There is a large proportion of CRC patients who are diagnosed with locally advanced disease with regional lymph node metastasis. Studying the mechanisms underlying the metastasis of CRC and identifying new clinical molecular markers have enabled further subclassification of patients and thereby a more accurate prediction of biological behavior and prognosis (2). To the best of our knowledge, there are a number of molecules that have been recently reported as CRC biomarker candidates (3). Among these, microRNA-133b (miR-133b) and TAp63 have attracted much attention due to accumulated evidence derived from basic and clinical studies supporting their clinical use (4-6).

miR-133b is one of the most significantly downregulated miRNAs in CRC (7). It has been reported to participate in the migration and invasion of certain types of cancer and is downregulated in tumor tissues, such as gastric (8), prostate (9) and CRC $(10,11)$. However, the mechanisms involved in miR-133b downregulation in cancer remain unclear. TAp63, a protein isoform of the p63 tumor-suppressor gene, is able to activate gene transcription and is closely related with metastasis $(12,13)$. In prostate and bladder cancer, TAp63 physically binds to the caspase- 1 promoter and directly regulates caspase-1 expression (14). However, TAp63 also directly regulates the expression of miRNAs, for example, 
miR-205 (15). We previously demonstrated that TAp63 expression leads to the transcriptional regulation of the expression of miR-133b (16). Therefore, we speculated that downregulation of TAp63 may be a mechanism involved in miR-133b dysregulation in CRC. A point mutation, or single base modification, is a type of mutation that causes a single nucleotide base substitution, insertion or deletion of genetic material. Point mutations have been proven to influence expression of many genes (17-19). In addition, miRNA gene point mutations have been reported in almost all types of cancer $(20,21)$. However, little is known regarding the possible impact of gene mutations on miR-133b expression.

In the present study, we aimed to understand whether the expression level of miR-133b is affected by TAp63 and miR-133b gene point mutations in CRC, and whether TAp63 and miR-133b expression can independently provide useful information in regards to the prediction of outcome in CRC.

\section{Materials and methods}

Materials. P63 rabbit monoclonal antibodies were purchased from Abcam (Cambridge, UK). GAPDH rabbit polyclonal antibody was purchased from ProteinTech Group, Inc. (Chicago, IL, USA).

Tissue specimens. A total of 38 pairs of formalin-fixed, paraffin-embedded (FFPE) sections of CRC tissues and non-cancerous tissues (NCTs) were obtained from the Pathology Department of the Third XiangYa Hospital of Central South University (Hunan, China) between October 2013 and June 2014. None of these patients had received radiotherapy or chemotherapy prior to surgery. The histopathological type and the stage of CRC were determined according to the criteria of the World Health Organization classification. All of the patients were staged using the US National Comprehensive Cancer Network (NCCN) Clinical Guidelines 2014. The present study was approved by the Ethics Committee of the Third XiangYa Hospital of Central South University. Patient consent was obtained both from the patients and the families of the patients.

RNA isolation and RT-qPCR. RT-qPCR was performed as previously described (16). Briefly, total RNA was isolated using an E.Z.N.A. Total RNA Kit II (Omega Bio-Tek Inc., Norcross, GA, USA). miRNA was isolated using an E.Z.N.A. PF miRNA Isolation kit (Omega Bio-Tek Inc.). RT-qPCR was performed using Real-Time Quantitative PCR SYBR-Green detection reagent (CoWin Biotech Co., Ltd., Beijing, China). miRNA RT-qPCR was performed using an All-in-One ${ }^{\mathrm{TM}}$ miRNA qRT-PCR Detection kit (GeneCopoeia, Rockville, MD, USA). Primers and the reaction conditions have been previously described (16). The relative expression of TAp63 was normalized using the $2^{-\Delta \Delta \mathrm{Ct}}$ method relative to GAPDH. The relative expression of miR-133b was normalized using the $2^{-\Delta \Delta \mathrm{Ct}}$ method relative to U6-snRNA. All PCR reactions were run in triplicate. Each sample was amplified in triplicate.

Western blotting. Western blot analysis was performed as previously described using primary antibodies (16). Briefly, cells were lysed in a lysis buffer and centrifuged at 14,000 x g at $4^{\circ} \mathrm{C}$ for $10 \mathrm{~min}$. The supernatants were collected and a BCA protein assay was performed. Total protein $(100 \mathrm{mg})$ was separated on a $10 \%$ polyacrylamide gel and transferred to polyvinylidene difluoride (PVDF) membranes (Invitrogen, Carlsbad, CA, USA). The membranes were blocked for $2 \mathrm{~h}$, and were then incubated with a primary antibody at $4^{\circ} \mathrm{C}$ overnight. After being washed with PBST, the membranes were incubated with an HRP-conjugated goat anti-rabbit IgG secondary antibody for $60 \mathrm{~min}$ at room temperature. The images were obtained using Kodak film (Shanghai, China). All experiments were performed in triplicate.

Immunohistochemical staining. Immunohistochemistry (IHC) was carried out using Dako EnVision System (Dako Diagnostics AG, Zug, Switzerland) following the manufacturer's protocol. For TAp63 protein, staining localized in the nuclei was considered positive. Images were captured using an Olympus microscope. Image-Pro Plus version 6.0 software (Media Cybernetics, Inc., Rockville, MD, USA) was used to assess the area and density of the dyed region, and the integrated optical density (IOD) value of the IHC section. The mean densitometry of the digital image (magnification, $\mathrm{x} 400$ ) was designated as representative TAp63 staining intensity (indicating the relative TAp63 expression level). The signal density of the tissue areas from five randomly selected fields were counted in a blinded manner and subjected to statistical analysis.

In situ hybridization (ISH). ISH was performed on CRC tissues. A locked nucleic acid (LNA) detection probe for miR133b, an LNA U6-positive and an LNA-negative control probe with a scramble sequence were used in this analysis. All probes were purchased from Auragene Bioscience Corporation, Inc. (Changsha, China). All probes were labeled with digoxigenin. After being washed with phosphate-buffered saline (PBS) and pre-hybridization, the slides were incubated with DIG-labeled LNA miR-133b, DIG-labeled LNA U6 probes (positive control) and DIG-labeled LNA scrambled sequence (negative control) at $49^{\circ} \mathrm{C}$ overnight. Washes were carried out at $49^{\circ} \mathrm{C}$, and then the slides were incubated with blocking solution. After PBS washing, the images were captured using an Olympus microscope. Image-Pro Plus version 6.0 software was used to assess the area and density of the dyed region and the IOD value of the ISH section.

Secondary structure prediction. The secondary structure of 446 bp pre-miR-133b sequences was predicted using RNAfold web server (http://nhjy.hzau.edu.cn/kech/swxxx/jakj/ dianzi/Bioinf4/miRNA/miRNA1.htm).

Statistical analysis. We analyzed the data from February 2015. All statistical calculations were performed using GraphPad Prism (GraphPad Prism software, version 6.01; GraphPad, San Diego, CA, USA) and Statistical Package for the Social Sciences (SPSS) PASW Statistics software, version 20 (SPSS, Inc., Chicago, IL, USA). A Mann-Whitney U test was used to compare differences between two groups. The correlation between TAp63 and miR-133b was determined by the Spearman's rank correlation test. The sensitivity and specificity in CRC tissues were calculated for each cut-off value. 
Table I. Association of the clinicopathological characteristics of the CRC cases with TAp63 and miR-133b mRNA expression.

\begin{tabular}{|c|c|c|c|c|c|c|c|}
\hline \multirow{2}{*}{$\begin{array}{l}\text { Clinicopathological } \\
\text { features }\end{array}$} & \multirow{2}{*}{$\begin{array}{l}\text { Cases } \\
(\mathrm{n}=38)\end{array}$} & \multicolumn{3}{|c|}{ TAp63 } & \multicolumn{3}{|c|}{$\operatorname{miR}-133 b$} \\
\hline & & $\operatorname{Low}^{\mathrm{a}}(\mathrm{n}=19)$ & $\operatorname{High}^{\mathrm{a}}(\mathrm{n}=19)$ & P-value & $\operatorname{Low}^{\mathrm{a}}(\mathrm{n}=19)$ & $\operatorname{High}^{\mathrm{a}}(\mathrm{n}=19)$ & P-value \\
\hline Mean age (years) & & $56.63 \pm 15.37$ & $58.95 \pm 10.51$ & 0.567 & $59.11 \pm 12.40$ & $56.47 \pm 13.86$ & 0.545 \\
\hline \multicolumn{8}{|l|}{ Tumor site } \\
\hline Left colon & 10 & 6 & 4 & 0.609 & 5 & 5 & 0.885 \\
\hline Right colon & 5 & 3 & 2 & & 3 & 2 & \\
\hline Rectum & 23 & 10 & 13 & & 11 & 12 & \\
\hline \multicolumn{8}{|l|}{ Tumor differentiation } \\
\hline Well & 6 & 4 & 2 & 0.549 & 3 & 3 & 0.888 \\
\hline Moderate & 27 & 12 & 15 & & 14 & 13 & \\
\hline Poor & 5 & 3 & 2 & & 2 & 3 & \\
\hline \multicolumn{8}{|l|}{ Staging } \\
\hline I & 5 & 2 & 3 & 0.679 & 3 & 2 & 0.731 \\
\hline II & 11 & 6 & 5 & & 6 & 5 & \\
\hline III & 18 & 8 & 10 & & 9 & 9 & \\
\hline IV & 4 & 3 & 1 & & 1 & 3 & \\
\hline \multicolumn{8}{|c|}{ Lymph node metastasis } \\
\hline No & 17 & 7 & 11 & $0.016^{\mathrm{b}}$ & 5 & 13 & $0.033^{\mathrm{b}}$ \\
\hline N1 & 12 & 4 & 7 & & 8 & 3 & \\
\hline $\mathrm{N} 2$ & 9 & 8 & 1 & & 6 & 3 & \\
\hline \multicolumn{8}{|l|}{ Distant metastasis } \\
\hline M0 & 34 & 15 & 19 & $0.034^{\mathrm{b}}$ & 15 & 19 & $0.034^{\mathrm{b}}$ \\
\hline M1 & 4 & 4 & 0 & & 4 & 0 & \\
\hline
\end{tabular}

${ }^{\text {aLow }}$ and high expression groups were determined by the median value of TAp63 and miR-133b mRNA in the 38 tumor tissue specimens. ${ }^{\mathrm{b}}$ Statistical significance $(\mathrm{P}<0.05)$.

The area under receiver-operating characteristic curve (AUC) was also estimated. The level of statistical significance was set at $\mathrm{P}<0.05$. All experiments were repeated three times.

\section{Results}

Clinicopathological characteristics of the patients. The clinical and histopathological features of the patients are summarized in Table I. In total 38 patients were included in the present study, which comprised 26 men and 12 women, with a combined age range of 55-83 years. Regarding the degree of cell differentiation, out of the 38 cases, 6 (15.79\%) were well-differentiated adenocarcinomas, 27 (71.05\%) were moderately differentiated and $5(13.16 \%)$ cases were poorly differentiated adenocarcinomas.

Regarding the evolutionary stage of the studied CRC cases, the majority of the patients were in the late disease stages: $5(11.16 \%)$ were diagnosed in stage I, $11(28.95 \%)$ in stage II, $18(47.37 \%)$ in stage III and $4(10.53 \%)$ in stage IV.

Expression of TAp63 is decreased in human CRC tissues. To study the expression of TAp63, we measured the mRNA and protein expression levels of TAp63 in 38 paired human CRC tissues and NCT samples using qRT-PCR, western blotting and IHC methods, respectively. Compared with the NCTs, the TAp63 mRNA expression level was significantly lower in $21(55.27 \%)$ tumor tissues (Fig. 1A, P<0.05). Consistent with the RT-qPCR results, a decrease of TAp63 protein expression was observed in the CRC tissues, compared with the NCTs (Fig. 1B, P<0.05). IHC also revealed that TAp63 was downregulated in the CRC tissues (Fig. 1C). Moreover, we evaluated the density of the staining signal of all 38 cases using the Image-Pro Plus 6.0 image analysis software. The mean density of cancerous tissues from all 38 cases was $0.005345 \pm 0.0005019$, while that of the adjacent tissue was $0.01483 \pm 0.0003317$. This result suggests a significantly lower level of TAp63 expression in CRC tissues compared to their NCTs (Fig. 1D, $\mathrm{P}<0.05$ ).

Downregulation of miR-133b is closely correlated with TAp63 expression. To study the relationship between TAp63 and miR-133b in human CRC, we assessed the expression levels of miR-133b in the 38 paired CRC and NCT samples using RT-qPCR and ISH. Compared with the NCTs, the miR-133b expression level was significantly lower in 31 (81.58\%) tumor tissues (Fig. 2A, P<0.05). ISH revealed that miR-133b was downregulated in the CRC tissues (Fig. 2B). Moreover, we measured the density of the staining signal in all 38 cases using 


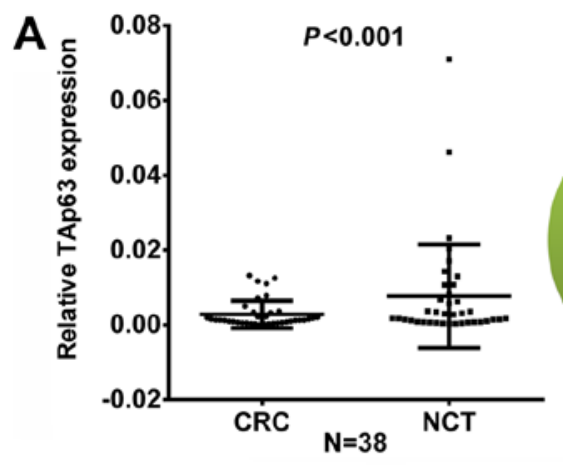

C

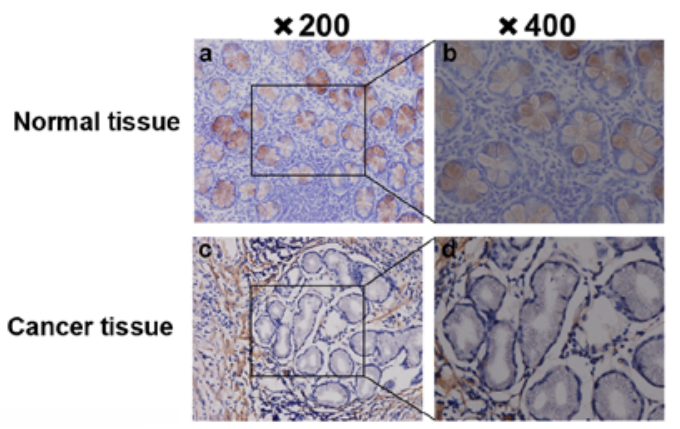

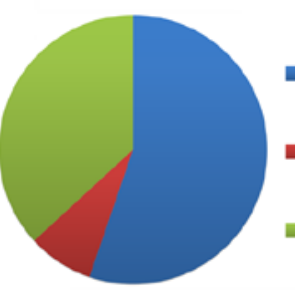

Underexpression $\mathbf{5 5 . 2 7 \%}$

Unchanged $7.89 \%$

- Overexpression $36.84 \%$
B

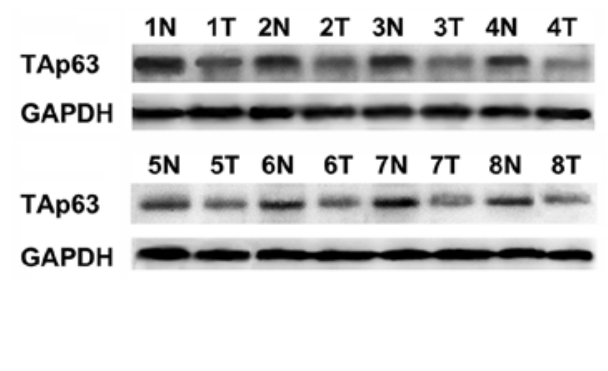

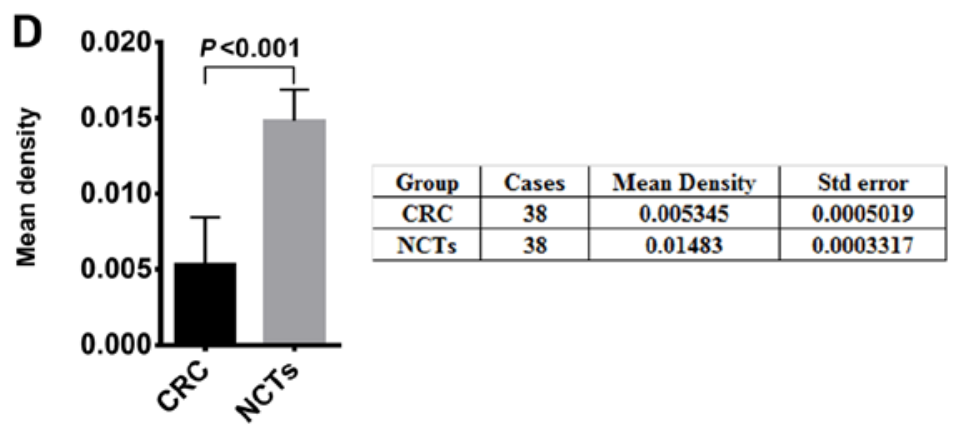

Figure 1. Expression of TAp63 in CRC patients. (A) Downregulated mRNA expression of TAp63 in CRC tissues as assessed by RT-qPCR (n=38; $\mathrm{P}<0.001)$. The mean expression of TAp63 is represented in the left scatter diagram and the expression distribution is summarized in the right pie chart. (B) Representative result of TAp63 protein expression in eight paired CRC and NCTs by IHC (T, tumor tissue; N, normal tissue). (C) IHC staining of CRC and NCTs from the same patient showed a low TAp63 expression. a, IHC staining of TAp63 in NCT (magnification, x200); b, IHC staining of TAp63 in NCT (magnification, x200); c, IHC staining of TAp63 in CRC tissue (magnification, x200); d, IHC staining of TAp63 in CRC tissue (magnification, x400). (D) TAp63 expression in CRC and NCTs. The mean density of TAp63 in tumor and normal tissues from 38 cases is illustrated in the left graph and is summarized in the table, on the right. A significant difference was detected between the mean density of TAp63 in CRC $(0.005345 \pm 0.0005019)$ and NCTs $(0.01483 \pm 0.0003317)$; P $<0.001$.
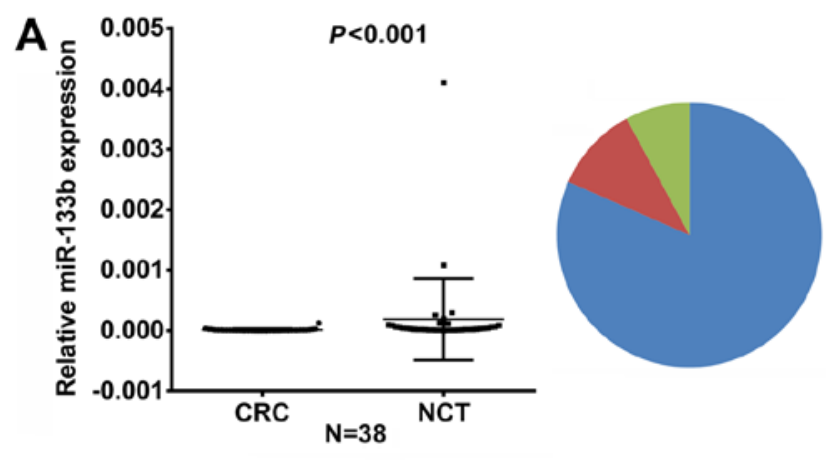

- Underexpression $\mathbf{8 1 . 5 8 \%}$

Unchanged $\mathbf{1 0 . 5 3 \%}$

- Overexpression $7.89 \%$

B

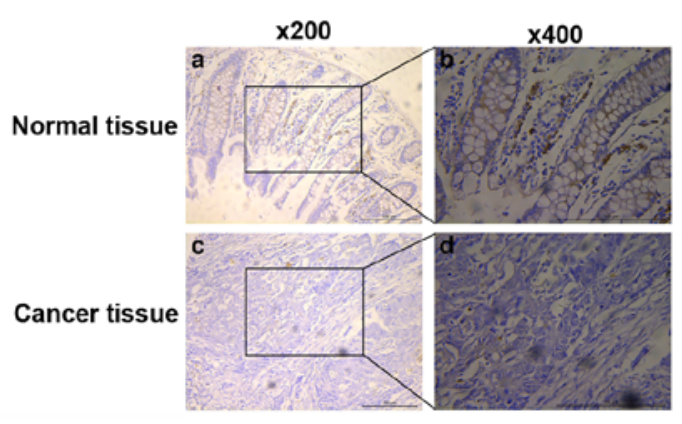

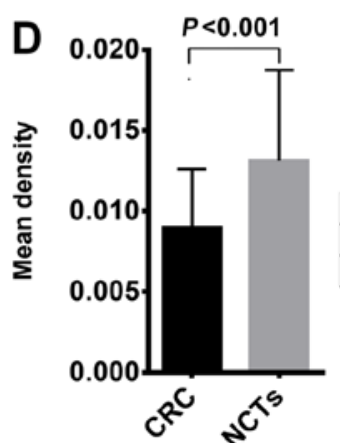

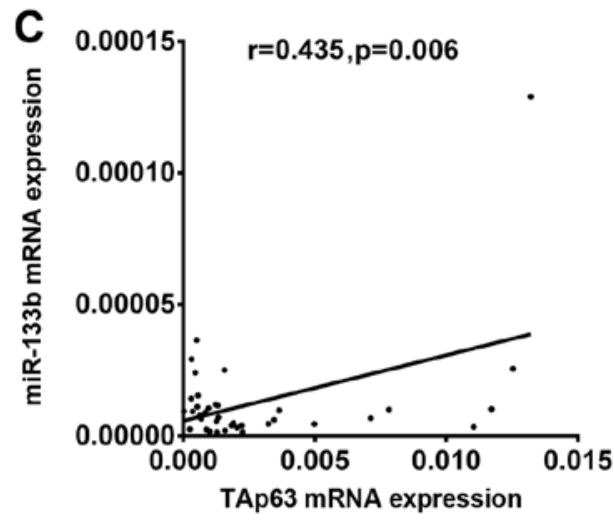

\begin{tabular}{c|c|c|c|} 
Group & Cases & Mean Density & Std error \\
\hline CRC & 38 & 0.009005 & 0.000585 \\
\hline NCTs & 38 & 0.01314 & 0.000909 \\
\hline
\end{tabular}

Figure 2. Expression of miR-133b in CRC patients. (A) Downregulated mRNA expression of miR-133b in CRC tissues as assessed by RT-qPCR (n=38; $\mathrm{P}<0.001)$. The mean expression of miR-133b is represented in the left scatter diagram and the expression distribution is summarized in the right pie chart. (B) ISH of miR-133b in CRC and NCTs from the same patient showed a low miR-133b expression. a, ISH of miR-133b in NCT (magnification, $\mathrm{x} 200$ ); $b$, ISH of miR-133b in NCT (magnification, $x 400$ ); c ISH of miR-133b in CRC tissue (magnification, $x 200$ ); $d$, ISH of miR-133b in CRC tissue (magnification, $x 400$ ). (C) Correlation of mRNA expression of TAp63 and miR-133b in 38 cases of CRC samples. Data revealed a positive correlation between TAp63 and miR-133b mRNA expression in the relevant CRC samples $(\mathrm{P}=0.006 ; \mathrm{r}=0.435)$. (D) miR-133b mRNA expression in CRC and NCTs. The mean density of miR-133b in tumor and normal tissues from 38 cases is illustrated in the left graph and summarized in the table, on the right. A significant difference was detected between the mean density of miR-133b in CRC $(0.009005 \pm 0.000585)$ and NCTs $(0.01314 \pm 0.000909) ; \mathrm{P}<0.001$. 
A

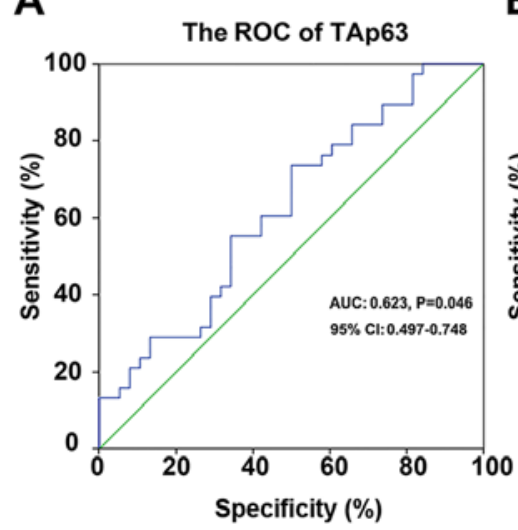

B

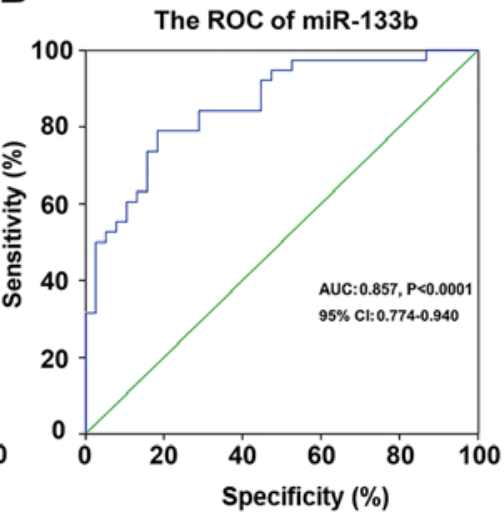

C The combined ROC of

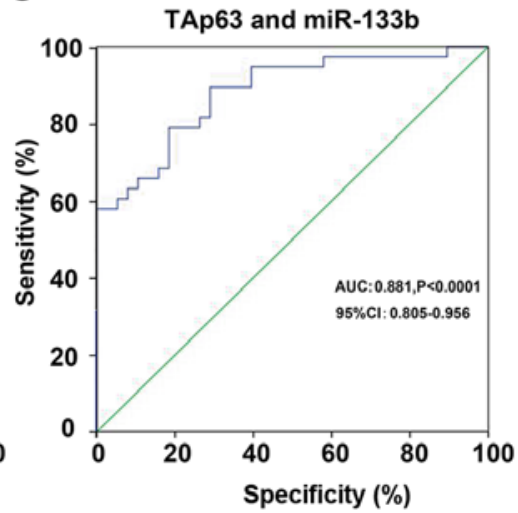

Figure 3. ROC curve analysis determines good sensitivity and specificity for the TAp63 and miR-133b level for discriminating CRC from non-tumor states of samples. (A) The mRNA levels of TAp63 yielded an AUC of 0.623 with $73.7 \%$ sensitivity and $50 \%$ specificity in discriminating CRC samples from NCTs. (B) The mRNA levels of miR-133b yielded an AUC of 0.857 with $78.9 \%$ sensitivity and $81.6 \%$ specificity in discriminating CRC samples from NCTs. (C) The combined mRNA levels of TAp63 and miR-133b yielded an AUC of 0.881 with $89.5 \%$ sensitivity and $71.1 \%$ specificity in discriminating CRC samples from NCTs.
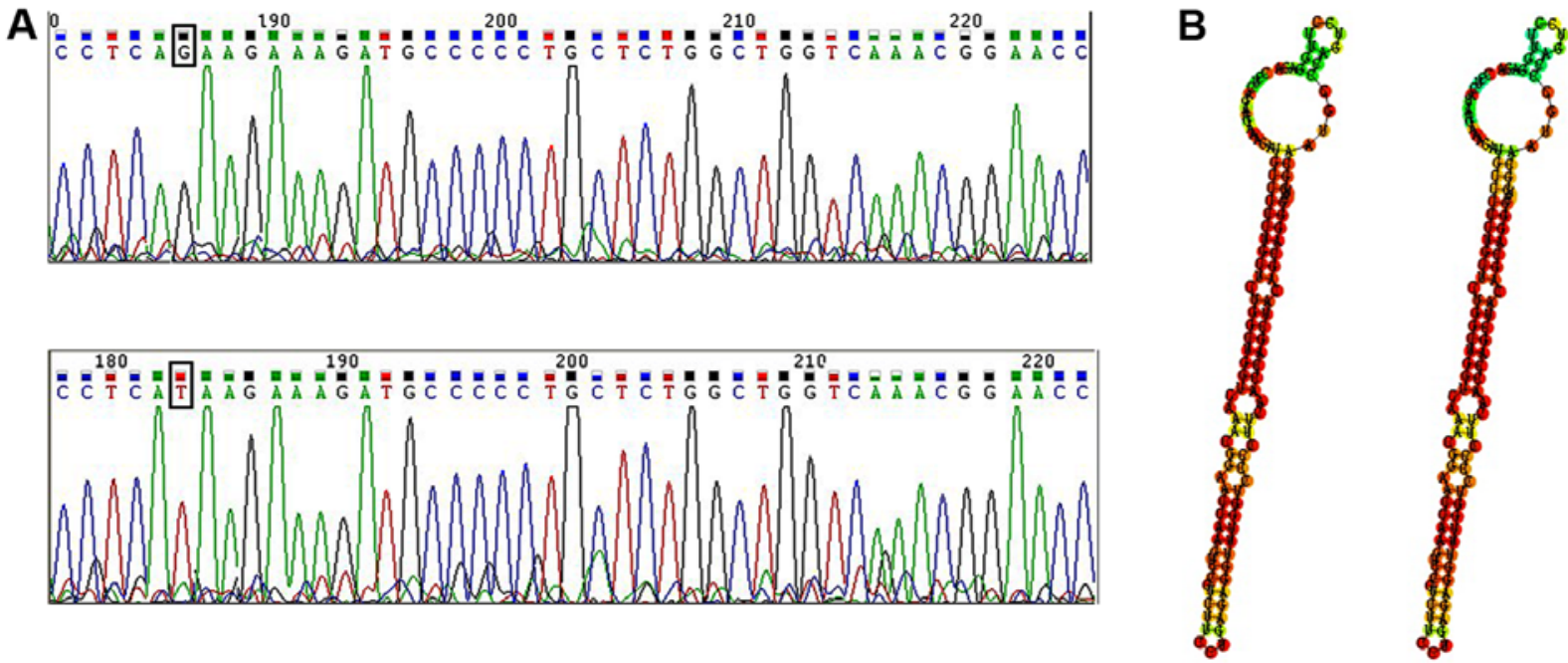

Figure 4. Point mutation in pri-miR-133b in CRC tissue. (A) DNA sequencing illustrating the genomic sequence of miR-133b DNA from CRC tissue. Nucleotides are indicated by capital letters. Note that the wild-type (wt) and mutant sequence are both present in the upper and lower genomic sequence, respectively. The base substitution, G-T, is located 6 bp from the 3' end of pre-miR-133b. (B) Predicted secondary structure of two genotype 446 bp pre-miR-133b sequences.

Image-Pro Plus 6.0 image analysis software. The mean density of cancerous tissues from all 38 cases was $0.009005 \pm 0.000585$, while that of the adjacent tissue was $0.01314 \pm 0.000909$. This result suggests a significantly lower level of miR-133b expression in the CRC tissues compared to their NCTs (Fig. 2D, $\mathrm{P}<0.05)$. In addition, the expression of miR-133b was found to be positively correlated with TAp63 (Fig. 2C, P<0.05).

Association of TAp63 and miR-133b with clinicopathological parameters. The association of TAp63 and miR-133b with various clinicopathological parameters of the CRC cases are listed in Table I. An inverse association was found between TAp63 expression and lymph node and distant metastases $(\mathrm{P}<0.05)$. miR-133b expression was also significantly correlated with lymph node and distant metastases. No statistically significant associations were found between the expression of TAp63 or miR-133b and other clinicopathological parameters ( $\mathrm{P}>0.05$ ).
By receiver operating characteristic (ROC) curve analysis, we proved that TAp63 and miR-133b are suitable predictors for CRC. The AUC of TAp63 for CRC was 0.623 [95\% confidence interval (CI), 0.497-0.748; $\mathrm{P}=0.046]$, with $73.7 \%$ sensitivity and $50 \%$ specificity, respectively (Fig. 3A). The AUC of miR-133b for CRC was 0.857 (95\% CI, 0.774-0.940, P<0.0001), with $78.9 \%$ sensitivity and $81.6 \%$ specificity, respectively (Fig. 3B). The combined AUC of TAp63 and miR-133b for CRC was 0.881 (95\% CI, 0.805-0.956, $\mathrm{P}<0.0001)$, with $89.5 \%$ sensitivity and $71.1 \%$ specificity, respectively (Fig. 3C).

miR-133b point mutation detection by DNA sequencing. To uncover whether there are point mutations in miR-133b DNA, we scanned 446 bp of the pre-miR-133b coding region. The results showed that only one patient had a single nucleotide change, $\mathrm{G}$ to $\mathrm{T}$, located $6 \mathrm{bp}$ from the $3^{\prime}$ end of pre-miR-133b (Fig. 4A). To further explore the function of the mutation site, we compared the miR-133b predicted secondary 
structure between the gene of the patient and the GenBank gene. As shown in Fig. 4B, the nucleotide change did not cause an apparent change in the stem-loop structure.

\section{Discussion}

Several studies have documented a link between the aberrant expression of TAp63 (22) and the pathogenesis/prognosis in several types of cancer $(23,24)$. However, thus far, the expression and clinical significance of TAp63 in colorectal cancer (CRC) have not been explored. In the present study, we evaluated the expression of TAp63 in CRC by RT-qPCR, western blotting and IHC, and analyzed its clinicopathological and prognostic significance in CRC patients. Our results showed that the TAp63 mRNA levels were significantly decreased in 21 (55.27\%) tumor tissues. In addition, an inverse association was found between TAp63 expression and lymph node and distant metastases. These results support an earlier hypothesis by Spiesbach et al (25) that TAp63 may be a cancer suppressor gene and suggest that TAp63 plays an important role in the tumorigenesis or progression of CRC. Moreover, compared with the NCTs, the miR-133b expression level was significantly lower in the $31(81.58 \%)$ tumor tissues and was positively correlated with TAp63. In addition, low expression of miR-133b was also significantly correlated with a higher metastasis of CRC, implying that a decrease in TAp63 and miR-133b expression may promote tumor metastasis.

Our group has previously shown that miR-133b promoter hypermethylation is upregulated in CRC tissues, and miR-133b promoter demethylation increased the expression of miR-133b (26). Moreover, TAp63 leads to the transcriptional regulation of the expression of miR-133b (16). However, whether the downregulation of TAp63 is one of the causes for the low expression of miR-133b in CRC has not been previously studied and in particular the role of gene point mutations in miR-133b expression has not been examined. In the present study we clearly showed that the expression of miR-133b is positively correlated with TAp63 and proposed the TAp63-alteration as a potential cause of miR-133b downregulation.

Notably, we found low expression of miR-133b in $31(81.58 \%)$ of the CRC tissues whereas low expression of TAp63 was found in 21 (55.27\%) of the CRC tissues. Obviously, reduced expression of the transcription factor is not the only factor that downregulated the expression of miR-133b in CRC. To the best of our knowledge, different types of miRNAs have been identified as being downregulated in breast (27), gastric (28) and colon cancer (29). In addition, some of them were systematically proved to be closely associated with genetic polymorphisms and mutations (30). During the present study, we found point mutations within the seed region of miR-133b in 1 patient. However, the predicting software showed that the point mutation did not impact the secondary structure of the pre-miR-133b. This result indicated that point mutations may not affect the expression level of miR-133b. Thus, in cancers, miR-133b downregulation tends to occur through miR-133b promoter hypermethylation and reduced expression of the transcription factor. Futhermore, the present study has some limitations. First, the sample size we screened for the sequence analysis was too small to find a missense mutation. Secondly, we did not verify the effectiveness of point mutations in CRC cells.

In conclusion, we demonstrated for the first time that the expression of TAp63 and miR-133b in CRC tissues is correlated with metastasis in clinical samples and speculated that downregulation of TAp63 may be one of the causes for the low expression of miR-133b in CRC. Our data suggest that TAp63 and miR-133b may function as potentially valuable diagnostic/prognostic biomarkers for CRC.

\section{Acknowledgements}

The present study was supported by the Science and Technology Project of Hunan Province (no. 2015SK20206), and the National Natural Science Foundation of China (no. 81172298).

\section{References}

1. Abu-Amero KK, Helwa I, Al-Muammar A, Strickland S, Hauser MA, Allingham RR and Liu Y: Screening of the seed region of MIR184 in keratoconus patients from Saudi Arabia. Biomed Res Int 2015: 604508, 2015.

2. Cristobal I, Madoz-Gurpide J, Martin-Aparicio E, Carames C, Aguilera O, Rojo F and Garcia-Foncillas J: Comment on 'TAp63 suppress metastasis via miR-133b in colon cancer cells'. Br J Cancer 111: 2369, 2014.

3. Kubisch J, Türei D, Földvári-Nagy L, Dunai ZA, Zsákai L, Varga M, Vellai T, Csermely $\mathrm{P}$ and Korcsmáros T: Complex regulation of autophagy in cancer - integrated approaches to discover the networks that hold a double-edged sword. Semin Cancer Biol 23: 252-261, 2013.

4. Shih IM and Kurman RJ: p63 expression is useful in the distinction of epithelioid trophoblastic and placental site trophoblastic tumors by profiling trophoblastic subpopulations. Am J Surg Pathol 28: 1177-1183, 2004.

5. Bassampour SA, Abdi R, Bahador R, Shakeri M, Torkaman A, Yahaghi E and Taheriazam A: Downregulation of miR-133b/ miR-503 acts as efficient prognostic and diagnostic factors in patients with osteosarcoma and these predictor biomarkers are correlated with overall survival. Tumour Biol: Aug 16, 2015 (Epub ahead of print).

6. Guzel E, Karatas OF, Semercioz A, Ekici S, Aykan S, Yentur S, Creighton $\mathrm{CJ}$, Ittmann $\mathbf{M}$ and Ozen $\mathrm{M}$ : Identification of microRNAs differentially expressed in prostatic secretions of patients with prostate cancer. Int J Cancer 136: 875-879, 2015.

7. Duan FT, Qian F, Fang K, Lin KY, Wang WT and Chen YQ: miR-133b, a muscle-specific microRNA, is a novel prognostic marker that participates in the progression of human colorectal cancer via regulation of CXCR4 expression. Mol Cancer 12: 164, 2013.

8. Zhao Y, Huang J, Zhang L, Qu Y, Li J, Yu B, Yan M, Yu Y, Liu B and Zhu Z: MiR-133b is frequently decreased in gastric cancer and its overexpression reduces the metastatic potential of gastric cancer cells. BMC Cancer 14: 34, 2014.

9. Li X, Wan X, Chen H, Yang S, Liu Y, Mo W, Meng D, Du W, Huang Y, Wu H, et al: Identification of miR-133b and RB1CC1 as independent predictors for biochemical recurrence and potential therapeutic targets for prostate cancer. Clin Cancer Res 20: 2312-2325, 2014.

10. Ellermeier C, Vang S, Cleveland K, Durand W, Resnick MB and Brodsky AS: Prognostic microRNA expression signature from examination of colorectal primary and metastatic tumors. Anticancer Res 34: 3957-3967, 2014.

11. Akçakaya P, Ekelund S, Kolosenko I, Caramuta S, Ozata DM, Xie H, Lindforss U, Olivecrona $\mathrm{H}$ and Lui WO: $m i R-185$ and $m i R-133 b$ deregulation is associated with overall survival and metastasis in colorectal cancer. Int J Oncol 39: 311-318, 2011.

12. Das RK, Anura A, Pal M, Bag S, Majumdar S, Barui A, Chakraborty C, Ray AK, Sengupta S, Paul RR, et al: Epithelio-mesenchymal transitional attributes in oral submucous fibrosis. Exp Mol Pathol 95: 259-269, 2013.

13. Zhang Y, Yan $\mathrm{W}$ and Chen X: P63 regulates tubular formation via epithelial-to-mesenchymal transition. Oncogene 33: 1548-1557, 2014. 
14. Celardo I, Grespi F, Antonov A, Bernassola F, Garabadgiu AV, Melino G and Amelio I: Caspase-1 is a novel target of p63 in tumor suppression. Cell Death Dis 4: e645, 2013.

15. Tucci P, Agostini M, Grespi F, Markert EK, Terrinoni A, Vousden KH, Muller PA, Dötsch V, Kehrloesser S, Sayan BS, et al: Loss of p63 and its microRNA-205 target results in enhanced cell migration and metastasis in prostate cancer. Proc Natl Acad Sci USA 109: 15312-15317, 2012.

16. Lin CW, Li XR, Zhang Y, Hu G, Guo YH, Zhou JY, Du J, Lv L, Gao K, Zhang Y, et al: TAp63 suppress metastasis via miR-133b in colon cancer cells. Br J Cancer 110: 2310-2320, 2014.

17. Gao QW, Hua LD, Wang J, Fan CX, Deng WY, Li B, Bian WJ Shao CX, He N, Zhou P, et al: A point mutation in SCN1A 5 genomic region decreases the promoter activity and is associated with mild epilepsy and seizure aggravation induced by antiepileptic drug. Mol Neurobiol: Mar 11, 2016 (Epub ahead of print).

18. Brooks MB, Catalfamo JL, MacNguyen R, Tim D, Fancher S and McCardle JAA: A TMEM16F point mutation causes an absence of canine platelet TMEM16F and ineffective activation and death-induced phospholipid scrambling. J Thromb Haemost 13 : 2240-2252, 2015.

19. Tan X, Wang H, Luo G, Ren S, Li W, Cui J, Gill HS, Fu SW and $\mathrm{Lu}$ Y: Clinical significance of a point mutation in DNA polymerase beta $(P O L B)$ gene in gastric cancer. Int J Biol Sci 11: 144-155, 2015

20. Lee HC, Yang CW, Chen CY and Au LC: Single point mutation of microRNA may cause butterfly effect on alteration of global gene expression. Biochem Biophys Res Commun 404: 1065-1069, 2011.

21. Palatnik JF, Wollmann H, Schommer C, Schwab R, Boisbouvier J, Rodriguez R, Warthmann N, Allen E, Dezulian T, Huson D, et al: Sequence and expression differences underlie functional specialization of Arabidopsis microRNAs miR159 and miR319. Dev Cell 13: 115-125, 2007.

22. Yamaki T, Suenaga Y, Iuchi T, Alagu J, Takatori A, Itami M, Araki A, Ohira M, Inoue M, Kageyama H, et al: Temozolomide suppresses $M Y C$ via activation of TAp63 to inhibit progression of human glioblastoma. Sci Rep 3: 1160, 2013.
23. Loljung L, Coates PJ, Nekulova M, Laurell G, Wahlgren M, Wilms T, Widlöf M, Hansel A and Nylander K: High expression of p63 is correlated to poor prognosis in squamous cell carcinoma of the tongue. J Oral Pathol Med 43: 14-19, 2014.

24. Bahnassy AA, Zekri AR, Salem SE, Abou-Bakr AA, Sakr MA, Abdel-Samiaa AG and Al-Bradei M: Differential expression of p53 family proteins in colorectal adenomas and carcinomas: Prognostic and predictive values. Histol Histopathol 29: 207-216, 2014.

25. Spiesbach K, Tannapfel A, Mössner J and Engeland K: TAp63gamma can substitute for p53 in inducing expression of the maspin tumor suppressor. Int J Cancer 114: 555-562, 2005.

26. Lv LV, Zhou J, Lin C, Hu G, Yi LU, Du J, Gao K and Li X: DNA methylation is involved in the aberrant expression of miR-133b in colorectal cancer cells. Oncol Lett 10: 907-912, 2015.

27. Tahiri A, Leivonen SK, Lüders T, Steinfeld I, Ragle Aure M, Geisler J, Mäkelä R, Nord S, Riis ML, Yakhini Z, et al: Deregulation of cancer-related miRNAs is a common event in both benign and malignant human breast tumors. Carcinogenesis 35: 76-85, 2014.

28. Ren J, Huang HJ, Gong Y, Yue S, Tang LM and Cheng SY: MicroRNA-206 suppresses gastric cancer cell growth and metastasis. Cell Biosci 4: 26, 2014

29. Zhang Z, Kim K, Li X, Moreno M, Sharp T, Goodheart MJ, Safe S, Dupuy AJ and Amendt BA: MicroRNA-26b represses colon cancer cell proliferation by inhibiting lymphoid enhancer factor 1 expression. Mol Cancer Ther 13: 1942-1951, 2014.

30. Chen AX, Yu KD, Fan L, Li JY, Yang C, Huang AJ and Shao ZM: Germline genetic variants disturbing the Let-7/LIN28 double-negative feedback loop alter breast cancer susceptibility. PLoS Genet 7: e1002259, 2011. 